\title{
Exchange between perverse and weight filtration for the Hilbert schemes of points of two surfaces
}

\author{
Mark Andrea A. de Cataldo*, Tamás Hausel ${ }^{\dagger}$ Luca Migliorini ${ }^{\ddagger}$
}

\begin{abstract}
We show that a natural isomorphism between the rational cohomology groups of the two zero-dimensional Hilbert schemes of $n$-points of two surfaces, the affine plane minus the axes and the cotangent bundle of an elliptic curve, exchanges the weight filtration on the first set of cohomology groups with the perverse Leray filtration associated with a natural fibration on the second set of cohomology groups. We discuss some associated hard Lefschetz phenomena.
\end{abstract}

\section{Contents}

1 Introduction 2

1.1 The main result . . . . . . . . . . . . . . . 2

1.2 Notation . . . . . . . . . . . . . . . . . . . 3

2 The Hilbert scheme of a surface and its cohomology groups 4

2.1 The decomposition theorem for the Hilbert-Chow map $\pi_{n}: S^{[n]} \rightarrow S^{(n)} \ldots$. 4

2.2 The MHS on $H^{*}\left(S^{[n]}, \mathbb{Q}\right) \ldots \ldots \ldots \ldots \ldots \ldots \ldots$

2.3 The map $\phi^{[n]}$ induced by a diffeomorphism $S_{2} \simeq S_{1} \ldots \ldots \ldots$

${ }^{*}$ Partially supported by N.S.A. and N.S.F.

${ }^{\dagger}$ Supported by a Royal Society University Research Fellowship

${ }^{\ddagger}$ Partially supported by PRIN 2007 project "Spazi di moduli e teoria di Lie" 
3 The surfaces $X$ and $Y$ and the filtrations ${ }_{\frac{1}{2}} \mathscr{W}_{Y^{[n]}}$ and $\mathscr{P}_{Y^{[n]}}$

3.1 The halved weight filtration $\frac{1}{2} \mathscr{W}_{Y^{[n]}}$ on $H^{*}\left(\left(\mathbb{C}^{*} \times \mathbb{C}^{*}\right)^{[n]}, \mathbb{Q}\right) \ldots \ldots$

3.2 Decomposition theorem for the Hitchin-like fibration $h_{n}: X^{[n]} \rightarrow \mathbb{C}^{(n)} \ldots$. 8

3.3 The perverse Leray filtration $\mathscr{P}_{X^{[n]}}$ on $H^{*}\left(X^{[n]}, \mathbb{Q}\right) \ldots \ldots \ldots$

4 The main result, relation with hard Lefschetz, and a speculation 12

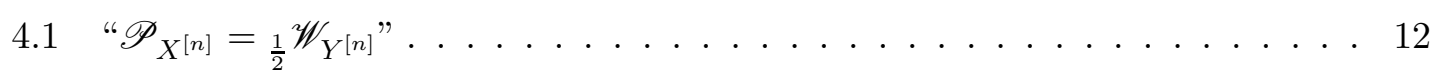

4.2 The curious hard Lefschetz $(\mathrm{CHL})$ for $H^{*}\left(\left(\mathbb{C}^{*} \times \mathbb{C}^{*}\right)^{[n]}, \mathbb{Q}\right) \ldots \ldots \ldots$

4.3 CHL on $Y^{[n]} \Leftrightarrow$ the HL on $E^{(\nu)} \Leftrightarrow$ RHL for $h_{n} \ldots \ldots \ldots$. . . . . . . 14

4.4 Speculating on where to find the exchange of filtrations . . . . . . . 16

\section{Introduction}

\subsection{The main result}

The theory of mixed Hodge structures endows the rational cohomology groups $H^{*}(Z, \mathbb{Q})$ of a complex algebraic variety $Z$ with the increasing weight filtration $\mathscr{W}_{Z}$. On the other hand, given a projective map $f: Z \longrightarrow Z^{\prime}$ from a nonsingular algebraic variety, the theory of perverse sheaves (with middle perversity) endows the rational cohomology groups $H^{*}(Z, \mathbb{Q})$ with the increasing perverse Leray filtration $\mathscr{P}_{Z}$ (see [11, for example). The filtration $\mathscr{P}_{Z}$ is analogous to the classical Leray filtration $\mathscr{L}_{Z}$ for the continuous map $f$. If $Z$ is nonsingular, then perverse Leray filtration is the counterpart in cohomology of a splitting, in the derived category of sheaves on $Z^{\prime}$, of the direct image complex $R f_{*} \mathbb{Q}_{Z}$ into a direct sum of shifted intersection cohomology complexes supported on closed subvarieties of $Z^{\prime}$. In general, the two filtrations differ and one has a strict inclusion $\mathscr{L}_{Z} \subseteq \mathscr{P}_{Z}$. The perverse Leray filtration admits a geometric description in terms of basic algebraic topology constructions; see the main result in [11].

In this paper, we give an example of the following remarkable phenomenon relating the weight and perverse filtrations for two distinct, yet related, varieties. Let $n \geq 0$ be an integer. Let $E$ be an elliptic curve and let $X:=T^{\vee}(E) \simeq E \times \mathbb{C}$ the total space of the cotangent bundle of $E$. The Hilbert scheme $X^{[n]}$ of $n$ points on $X$ is a $2 n$-dimensional nonsingular variety admitting a proper flat map $h_{n}: X^{[n]} \rightarrow \mathbb{C}^{(n)} \simeq \mathbb{C}^{n}$ of relative dimension $n$ onto the $n$-th symmetric product $\mathbb{C}^{(n)}$ of $\mathbb{C}$. Let $Y:=\mathbb{C}^{*} \times \mathbb{C}^{*}$ and $Y^{[n]}$ be the 
corresponding Hilbert scheme of $n$-points on $Y$. The weights of the mixed Hodge structure on $H^{*}\left(Y^{[n]}, \mathbb{Q}\right)$ are even, so that it makes sense to define the halved weight filtration ${ }_{\frac{1}{2}} \mathscr{W}_{Y^{[n]}, k}:=\mathscr{W}_{Y^{[n]}, 2 k}$ on $H^{*}\left(Y^{[n]}, \mathbb{Q}\right)$.

The main result of this paper is Theorem 4.1.1 which establishes that there is a natural isomorphism of graded vector spaces $\phi^{[n]}: H^{*}\left(X^{[n]}, \mathbb{Q}\right) \simeq H^{*}\left(Y^{[n]}, \mathbb{Q}\right)$ that exchanges the perverse Leray filtration for the map $h_{n}$ with the halved weight filtration defined above:

$$
\phi^{[n]}\left(\mathscr{P}_{X^{[n]}}\right)={ }_{\frac{1}{2}} \mathscr{W}_{Y^{[n]}} .
$$

In words, the isomorphism $\phi^{[n]}$ sends a class in $\mathscr{P}_{X^{[n]}, p}$ to a class of type $(p, p)$ for $\mathscr{W}_{Y^{[n]}}$. Theorem4.3.2 relates the hard Lefshcetz theorem on the products of symmetric products of the curve $E$ with the relative hard Lefschetz theorem for the map $h_{n}$ and with a "curious" hard Lefschetz theorem on the cohomology of $Y^{[n]}$.

In the paper [3], we have proved that the same "exchange of filtration" phenomenon appears in the case of the moduli space of degree one and rank two Higgs bundles on a curve of genus $g \geq 2$, endowed with the associated Hitchin map, and of the associated twisted character variety. See 4.4 for a precise statement of this result.

The example dealt with in this paper presents a striking difference with respect to the one treated in [3]. In the latter case, due to Nĝ's support theorem, most of the perverse sheaves showing up in the decomposition theorem are supported on all of the target space of the Hitchin map. On the other hand, in the case treated here, every stratum in $\mathbb{C}^{(n)}$ of the map $h_{n}: X^{[n]} \rightarrow \mathbb{C}^{(n)}$ contributes several perverse sheaves showing up in the decomposition theorem.

At the moment, we cannot explain the exchange of filtration phenomena described above, beyond the fact that we can observe them. In 4.4 we discuss some properties shared by the example considered in this paper and the one treated in [3], and we speculate on the possibility of a more general statement regarding the phenomenon of exchange of filtrations.

\subsection{Notation}

We work over the field of complex numbers $\mathbb{C}$ and with singular cohomology with rational coefficients $\mathbb{Q}$. The results hold with no essential changes over any algebraically closed field and with $\mathbb{Q}_{\ell}$-adic cohomology. A variety is a separated scheme of finite type over $\mathbb{C}$.

We employ freely the language of derived categories, perverse sheaves and the decomposition theorem as well as the language of Deligne's mixed Hodge structures (MHS); 
the reader may consult [1], the survey [10] and the textbooks [14, 21, 20, 24]. For the convenience of the reader we summarize our notation and terminology below.

Given a variety $Z$, we work with the full subcategory $D_{Z}$ of the derived category of the category of sheaves of rational vector spaces on $Z$ given by those bounded complexes $K$ on $Z$ whose cohomology sheaves $\mathcal{H}^{i}(K)$ on $Z$ are constructible; a sheaf on $Z$ is constructible if there is a partition $Z=\coprod Z_{a}$ of $Y$ given by locally closed subvarieties such that the restriction $F_{\mid Z_{a}}$ is locally constant for every $a$. We denote the $i$-th perverse cohomology sheaf of a complex $K$ on $Z$ by $\mathcal{H}^{i}(K)$; it is a perverse sheaf on $Z$. Given a map $f: Z \rightarrow Z^{\prime}$ of algebraic varieties, we denote the derived direct image functor $R f_{*}$ simply by $f_{*}$ and the $i$-th direct image functor by $R^{i} f_{*}$.

A filtration $F$ on a vector space is a finite increasing filtration $\ldots \subseteq F_{i} V \subseteq F_{i+1} V \subseteq \ldots$; finite means that $F_{i} V=\{0\}$ for $i \ll 0$ and $F_{i}=V$ for $i \gg 0$. A filtration $F$ on $V$ has type $[a, b]$ if $F_{a-1} V=\{0\}$ and $F_{b} V=V$.

Given a variety $Z$, the weight filtration on the cohomology groups $H^{d}(Z, \mathbb{Q})$ is denoted by $\mathscr{W}_{Z}$. A map $f: Z \rightarrow Z^{\prime}$ endows the cohomology groups $H^{d}(Z, \mathbb{Q})$ with two distinct filtrations, the Leray filtration $\mathscr{L}_{Z}$ and the perverse Leray filtration $\mathscr{P}_{Z}$.

In this paper, we are concerned with the Hilbert schemes of $n$ points $X^{[n]}$ and $Y^{[n]}$ associated with the two complex surfaces $X:=T^{\vee} E \simeq E \times \mathbb{C}$, the total space of the cotangent bundle of an elliptic curve $E$, and $Y:=\mathbb{C}^{*} \times \mathbb{C}^{*}$. We shall consider a certain natural proper map $h_{n}: X^{[n]} \rightarrow \mathbb{C}^{(n)}$.

\section{The Hilbert scheme of a surface and its cohomology groups}

\subsection{The decomposition theorem for the Hilbert-Chow map $\pi_{n}: S^{[n]} \rightarrow$ $S^{(n)}$}

Let $S$ be a nonsingular connected complex analytic surface $S$ and $n \geq 0$ be a non-negative integer. We refer the reader to [4, 15, 7, 15, 23] for background and references on Hilbert schemes of surfaces.

We denote by $S^{(n)}:=S^{n} / \mathfrak{S}_{n}$ the $n$-th symmetric product of $S$, i.e. the quotient of $S^{n}$ by the obvious action of the $n$-th symmetric group. A partition of $\nu=\left\{\nu_{1}, \ldots, \nu_{l}\right\}$ of $n$ is an unordered collection of positive integers such that $\nu_{1}+\ldots+\nu_{l}=n$; the integer $l=l(\nu)$ is called the length of $\nu$. A point $x \in S^{(n)}$ gives rise to a partition $\nu=\nu(x)$, for $x$ admits a unique representation as a formal sum $\nu_{1} s_{1}+\ldots+\nu_{l} s_{l}$, with $\nu_{i}$ positive integers adding up to $n$, and $s_{i} \in S$ distinct. The subset $S_{\nu}^{(n)} \subseteq S^{(n)}$ of points yielding 
the same partition $\nu$ is a locally closed, irreducible, nonsingular subvariety of $S^{(n)}$ and we have that the symmetric product $S^{(n)}$ is the disjoint union over the set of partitions on $n$ of these subvarieties: $S^{(n)}=\coprod_{\nu} S_{\nu}^{(n)}$. A partition $\nu$ gives rise to a new variety $S^{(\nu)}$ as follows: represent the partition $\nu$ as a symbol $1^{a_{1}} 2^{a_{2}} \cdots n^{a_{n}}$, where $a_{i}$ is the number of times $i$ appears in $\nu$; the $a_{i} \geq 0$, the length $l(\nu)=\sum a_{i}$ and $n=\sum_{i} i a_{i}$; finally, define $S^{(\nu)}:=\prod_{i} S^{\left(a_{i}\right)}$ to be the indicated product of symmetric product of $S$. If we define, $\mathfrak{S}_{\nu}:=\prod \mathfrak{S}_{a_{i}}$, then $S^{(\nu)}=S^{l(\nu)} / \mathfrak{S}_{\nu}$. There is a natural finite map $r^{(\nu)}: S^{(\nu)} \rightarrow S^{(n)}$ with image the closure $\overline{S_{\nu}^{(n)}}$ and the resulting map $S^{(\nu)} \rightarrow \overline{S_{\nu}^{(n)}}$ is the normalization of the image.

The Hilbert scheme $S^{[n]}$ of zero-dimensional length $n$ subschemes of $S$ is a connected complex manifold of dimension $2 n$ and, if $S$ is algebraic, then so is $S^{[n]}$. There is the $n$-th Hilbert-Chow map $\pi_{n}: S^{[n]} \rightarrow S^{(n)}$ sending a scheme to its support, counting multiplicities; this map is proper and it is a resolution of singularities of the symmetric product.

In view of [7], $\S 2.5$, by using the correspondences in $S^{(\nu)} \times{ }_{S^{(n)}} S^{[n]}$ inside $S^{(\nu)} \times S^{[n]}$ the decomposition theorem for the map $\pi_{n}$ yields a canonical isomorphism in the category $D_{S^{(n)}}$

$$
\gamma_{S}^{[n]}:=\sum_{\nu} \gamma_{S}^{(\nu)}: \bigoplus_{\nu} r_{*}^{(\nu)} \mathbb{Q}_{S^{(\nu)}}[2 l(\nu)] \stackrel{\simeq}{\longrightarrow} \pi_{*} \mathbb{Q}_{S}[n][2 n]
$$

\subsection{The MHS on $H^{*}\left(S^{[n]}, \mathbb{Q}\right)$}

If $S$ is algebraic, then, by using the compatibility (see [8]) with MHS of the constructions leading to the isomorphism (11), we obtain a canonical isomorphism of MHS (recall that a Tate twist in cohomology $(-i)$ increases the weights by $2 i)$ :

$$
\gamma_{S}^{[n]}=\sum \gamma_{S}^{(\nu)}: \bigoplus_{\nu}\left(H^{*-2[n-l(\nu)]}\left(S^{(\nu)}, \mathbb{Q}\right)(l(\nu)-n)\right) \stackrel{\simeq}{\longrightarrow} H^{*}\left(S^{[n]}, \mathbb{Q}\right) .
$$

The fact that the two sides of (2) are isomorphic has been first proved in [16] by using the theory of mixed Hodge modules.

Given a partition $\nu$ of $n$, consider the mixed Hodge substructure

$$
H_{\nu}^{*}\left(S^{[n]}, \mathbb{Q}\right):=\operatorname{Im} \gamma_{S}^{(\nu)} \simeq H^{*-2[n-l(\nu)]}\left(S^{(\nu)}, \mathbb{Q}\right)(l(\nu)-n)
$$

so that the isomorphism of MHS (2) now reads as the internal direct sum decomposition

$$
H^{*}\left(S^{[n]}, \mathbb{Q}\right)=\bigoplus_{\nu} H_{\nu}^{*}\left(S^{[n]}, \mathbb{Q}\right) .
$$




\subsection{The map $\phi^{[n]}$ induced by a diffeomorphism $S_{2} \simeq S_{1}$}

The canonical isomorphism (2) has the following simple consequence. Let $S_{1}$ and $S_{2}$ be two nonsingular surfaces and

$$
\phi: H^{*}\left(S_{1}, \mathbb{Q}\right) \simeq H^{*}\left(S_{2}, \mathbb{Q}\right)
$$

be an isomorphism of graded vector spaces. By taking tensor products and invariants, the map $\phi$ induces, for every partition $\nu$, an isomorphism of graded vector spaces $\phi^{(\nu)}$ : $H^{*}\left(S_{1}^{(\nu)}, \mathbb{Q}\right) \simeq H^{*}\left(S_{2}^{(\nu)}, \mathbb{Q}\right)$.

By using the isomorphisms (1), we define the map

$$
\phi^{[n]}:=\left(\gamma_{S_{2}}^{[n]}\right) \circ\left(\sum_{\nu} \phi^{(\nu)}\right) \circ\left(\gamma_{S_{1}}^{[n]}\right)^{-1}: H^{*}\left(S_{1}^{[n]}, \mathbb{Q}\right) \simeq H^{*}\left(S_{2}^{[n]}, \mathbb{Q}\right)
$$

which is an isomorphisms of graded vector spaces.

If the surfaces $S_{i}$ are algebraic and $\phi$ is an isomorphism of MHS, then so is (6). However, in this paper we use this set-up in the case: $S_{1}=E \times \mathbb{C}$ ( $E$ an elliptic curve) and $S_{2}=\mathbb{C}^{*} \times \mathbb{C}^{*}$ and $\phi=\Phi^{*}$, where $\Phi: S_{2} \simeq S_{1}$ is a diffeomorphism. In this case, due to the incompatibility of the weights, $\phi$ and $\phi^{[n]}$ cannot be isomorphisms of MHS.

It is likely that the results in [25] imply that if we have a diffeomorphism $S_{2} \simeq S_{1}$ of

nonsingular algebraic surfaces, then there is a diffeomorphism $S_{2}^{[n]} \simeq S_{1}^{[n]}$. At present, we do not know this and we do not need it here.

\section{The surfaces $X$ and $Y$ and the filtrations ${ }_{\frac{1}{2}} \mathscr{W}_{Y^{[n]}}$ and $\mathscr{P}_{Y^{[n]}}$}

For the remainder of the paper, we fix $n \geq 0$, an elliptic curve $E$ and we set

$$
Y:=\mathbb{C}^{*} \times \mathbb{C}^{*}, \quad X:=T^{\vee} E \simeq E \times \mathbb{C},
$$

i.e. $X$ is the total space of the cotangent (canonical) bundle of $E$. The isomorphism above is well-defined up to multiplication by a non-zero scalar.

The two surfaces $X$ and $Y$ are noncanonically diffeomorphic: choose $E$ to be $\mathbb{C} / \Gamma$ where $\Gamma$ is the lattice of Gaussian integers; then use polar coordinates to identify $X$ and $Y$. Let $\Phi: Y \simeq X$ be any diffeomorphism and set $\phi:=\Phi^{*}: H^{*}(X, \mathbb{Q}) \simeq H^{*}(Y, \mathbb{Q})$. We are in the situation of $\$ 2.3$, (15) so that, for every $n \geq 0$, we obtain the linear isomorphism (6) of graded vector spaces

$$
\phi^{[n]}: H^{*}\left(X^{[n]}, \mathbb{Q}\right) \stackrel{\simeq}{\longrightarrow} H^{*}\left(Y^{[n]}, \mathbb{Q}\right) .
$$


As it was observed in $₫ 2.3$, for $n \geq 1$, the two sides are never isomorphic as MHS. In particular, (7) does not preserve the weight filtrations.

Let us remark that each $H^{d}\left(X^{[n]}, \mathbb{Q}\right)$ is a pure Hodge structure of weight $d$. Since $H^{*}(X, \mathbb{Q}) \simeq H^{*}(E, \mathbb{Q})$ is an isomorphism of MHS, we have that the same is true for $H^{*}\left(X^{(\nu)}, \mathbb{Q}\right) \simeq H^{*}\left(E^{(\nu)}, \mathbb{Q}\right)$ for every partition $\nu$ of $n$. In view of the splitting of MHS (4), we have the following canonical isomorphism of MHS

$$
H^{*}\left(X^{[n]}, \mathbb{Q}\right) \stackrel{(4)}{=} \bigoplus_{\nu} H_{\nu}^{*}\left(X^{[n]}, \mathbb{Q}\right) \simeq \bigoplus_{\nu} H^{*-2[n-l(\nu)]}\left(E^{(\nu)}, \mathbb{Q}\right)(l(\nu)-n) .
$$

Since each $H^{d}\left(E^{(\nu)}, \mathbb{Q}\right)$ is pure of weight $d$, we conclude that each $H^{d}\left(X^{[n]}, \mathbb{Q}\right)$ is pure of weight $d$ as well. In particular, the weight filtration $\mathscr{W}_{X^{[n]}}$ on $H^{*}\left(X^{[n]}, \mathbb{Q}\right)$ is simply the filtration by cohomological degree and this should be contrasted with Proposition 3.1.2.

\subsection{The halved weight filtration $\frac{1}{2} \mathscr{W}_{Y^{[n]}}$ on $H^{*}\left(\left(\mathbb{C}^{*} \times \mathbb{C}^{*}\right)^{[n]}, \mathbb{Q}\right)$}

In this section, we first compute the MHS on $H^{*}\left(Y^{[n]}, \mathbb{Q}\right)$ and determine the weight filtration $\mathscr{W}_{Y^{[n]}}$ on $H^{*}\left(Y^{[n]}, \mathbb{Q}\right)$. We then observe that $\mathscr{W}_{Y^{[n]}}$ has no odd weights so that we can define the halved weight filtration $\frac{1}{2} \mathscr{W}_{Y^{[n]}, k}:=\mathscr{W}_{2 k}$ on $H^{*}\left(Y^{[n]}, \mathbb{Q}\right)$ by simply halfing the weights.

Recall that: an MHS is of Hodge-Tate type if the odd graded pieces of the weight filtration are zero and every even graded piece $\operatorname{Gr}_{2 p}^{\mathscr{W}}$ is of pure type $(p, p)$; an MHS is split of Hodge-Tate type if it is isomorphic to a direct sum of pure MHS of Hodge-Tate type.

Lemma 3.1.1 For every partition $\nu$ of $n$, the natural $M H S$ on $H^{*}\left(Y^{(\nu)}, \mathbb{Q}\right)$ is split of Hodge-Tate type and, more precisely,

$$
\begin{gathered}
H^{d}\left(Y^{(\nu)}, \mathbb{Q}\right) \text { is pure of weight } 2 d \text { and Hodge-type }(d, d), \\
0=\mathscr{W}_{2 d-1} \subseteq \mathscr{W}_{2 d}=H^{d}\left(Y^{(\nu)}, \mathbb{Q}\right) .
\end{gathered}
$$

Proof. Since $H^{d}\left(\mathbb{C}^{*}, \mathbb{Q}\right)$ has type $(d, d)$, for $d=0,1$, and it is trivial otherwise, the statement follows from the Künneth isomorphism and the naturality of the mixed Hodge structure for the inclusion $H^{d}\left(Y^{(\nu)}, \mathbb{Q}\right) \subseteq H^{d}\left(Y^{l(\nu)}, \mathbb{Q}\right)$ coming from the quotient map $Y^{l(\nu)} \longrightarrow Y^{l(\nu)} / \mathfrak{S}_{\nu}=Y^{(\nu)}$.

The following proposition is an immediate consequence of Lemma 3.1.1 and of the equality of MHS (41). 
Proposition 3.1.2 The natural mixed Hodge structure on $H^{*}\left(Y^{[n]}, \mathbb{Q}\right)$ is split of HodgeTate type. More precisely, in terms of the decomposition (4), we have:

$$
\mathscr{W}_{2 k} H^{d}\left(Y^{[n]}, \mathbb{Q}\right)=\bigoplus_{d-(n-l(\nu)) \leq k} H_{\nu}^{d}\left(Y^{[n]}, \mathbb{Q}\right), \quad \mathscr{W}_{2 k}=\mathscr{W}_{2 k+1}
$$

Proposition 3.1 .2 allows us to define the halved weight filtration $\frac{1}{2}_{Y^{[n]}}$ by setting

$$
{ }_{\frac{1}{2}} \mathscr{W}_{Y^{[n]}, k}:=\mathscr{W}_{Y^{[n]}, 2 k}
$$

The halved weight filtration $\frac{1}{2} \mathscr{W}_{Y^{[n]}}$ on $H^{*}\left(Y^{[n]}, \mathbb{Q}\right)$ has type $[0,2 n]$.

\subsection{Decomposition theorem for the Hitchin-like fibration $h_{n}: X^{[n]} \rightarrow \mathbb{C}^{(n)}$}

Let $p: X \rightarrow \mathbb{C}$ be the induced projection. Recall the notation in 92.2 , We have the commutative diagram

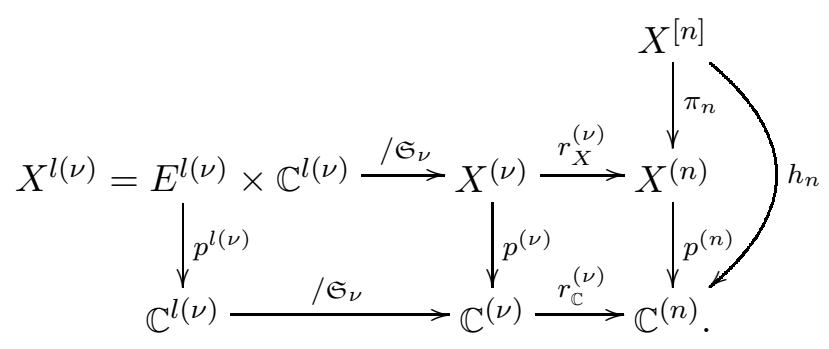

The maps $p^{l(\nu)}$ and $p^{(\nu)}$ are of relative dimension $l(\nu)$ and the map $p^{(n)}$ is of relative dimension $n$. In particular, note that

$$
\operatorname{dim}\left\{p^{(n)^{-1}}\left(\mathbb{C}_{\nu}^{(n)}\right)\right\}=l(\nu)+n, \quad \operatorname{dim}\left\{p^{(\nu)^{-1}}\left(\mathbb{C}_{\nu}^{(\nu)}\right)\right\}=2 l(\nu) .
$$

The fiber of $p^{(\nu)}$ over the general point of $\mathbb{C}^{(\nu)}$ is isomorphic to $E^{l(\nu)}$. All the other fibers are isomorphic to quotients of $E^{l(\nu)}$ under the action of suitable, not necessarily normal, subgroups groups of the finite group $\mathfrak{S}_{\nu}$. The fibers over the points in the small diagonal in $\mathbb{C}^{(\nu)}$ are all isomorphic to $E^{(\nu)}=E^{l(\nu)} / \mathfrak{S}_{\nu}$ so that, by the compatibility with MHS of Grothendieck's theorem on the rational cohomology of quotient varieties, we have a canonical isomorphism of MHS

$$
H^{*}\left(E^{(\nu)}, \mathbb{Q}\right)=H^{*}\left(E^{l(\nu)}, \mathbb{Q}\right)^{\mathfrak{S}_{\nu}}
$$


The map $h_{n}: X^{[n]} \rightarrow \mathbb{C}^{(n)}$ is projective of relative dimension $n=\frac{1}{2} \operatorname{dim} X^{[n]}=$ $\operatorname{dim} \mathbb{C}^{(n)}$, flat by 22 , Corollary to Theorem 23.1, and, as it is observed above, it has general fiber the Abelian variety $E^{l(\nu)}$.

Remark 3.2.1 We say that $h_{n}$ is a Hitchin-type map because of the analogy it presents with the Hitchin map associated with the moduli of Higgs bundles on a curve, where the dimensions of domain $M$, target $A$ and fibers $F$ are related as above: $\operatorname{dim} M=2 \operatorname{dim} A=$ $2 \operatorname{dim} F$ and also because our main result Theorem 4.1.1 is analogous to the main result of [3], which deals with rank two Higgs bundles of odd degree on a curve.

Due to the commutativity of the diagram (8) and to the functoriality of derived pushforwards applied to $h_{n}=p^{(n)} \circ \pi_{n}$, the decomposition theorem (1) for the map $\pi_{n}$ implies that we have natural isomorphisms

$$
\bigoplus_{\nu} r_{\mathbb{C} *}^{(\nu)} p_{*}^{(\nu)} \mathbb{Q}_{X^{(\nu)}}[2 l(\nu)] \longrightarrow \bigoplus_{\nu} p_{*}^{(n)} r_{X_{*}}^{(\nu)} \mathbb{Q}_{X^{(\nu)}}[2 l(\nu)] \stackrel{\simeq}{\longrightarrow} h_{n *} \mathbb{Q}_{X^{[n]}}[2 n]
$$

By applying Grothendieck theorem on the invariant part of push-forwards under a quotient map under a finite group action, and by recalling that $p^{l(\nu)}$ is a projection map, we get a canonical isomorphism

$$
p_{*}^{(\nu)} \mathbb{Q}_{X^{(\nu)}}=\left(p_{*}^{l(\nu)} \mathbb{Q}_{X^{(\nu)}}\right)^{\mathfrak{S}_{\nu}}=\bigoplus_{i=0}^{2 l(\nu)}\left(R^{i} p_{*}^{l(\nu)} \mathbb{Q}\right)^{\mathfrak{S}_{\nu}}[-i] .
$$

We thus get the distinguished splitting isomorphism in the category $D_{\mathbb{C}^{(n)}}$

$$
\Gamma_{X}^{[n]}: \bigoplus_{\nu} \bigoplus_{i=0}^{2 l(\nu)}\left\{\left[r_{\mathbb{C} *}^{(\nu)}\left(R^{i} p_{*}^{l(\nu)} \mathbb{Q}\right)^{\mathfrak{S}_{\nu}}\right][l(\nu)]\right\}[-(i-l(\nu))] \stackrel{\simeq}{\longrightarrow} h_{*} \mathbb{Q}_{X^{[n]}}[2 n]
$$

Since every $r^{(\nu)}$ is finite, every direct summand in square brackets is an ordinary sheaf (not just a complex). Moreover, since the functors $r_{*}^{(\nu)}$ are $t$-exact, every summand in curly brackets is a perverse sheaf, in fact an intersection cohomology complex with twisted coefficients supported on $\overline{\mathbb{C}_{\nu}^{(n)}} \subseteq \mathbb{C}^{(n)}$.

It follows that (10) "is" the decomposition theorem for the map $h_{n}$ in the sense that we decomposed the right-hand-side as direct sum of shifted intersection cohomology complexes supported on $\mathbb{C}^{(n)}$. We note that, unlike the general statement of the decomposition theorem, we have obtained (10) as a distinguished isomorphism.

In order to simplify the notation, we set

$$
R_{\nu}^{i}:=r_{\mathbb{C} *}^{(\nu)}\left(R^{i} p_{*}^{l(\nu)} \mathbb{Q}\right)^{\mathfrak{S}_{\nu}}
$$


For our purposes, it is convenient to re-write (10) in the following two different ways, where the former emphasizes the perverse-sheaf-nature of the summands, and the latter emphasizes the ordinary-sheaf-nature of the summands. One merely needs to apply the appropriate shift and re-organize the terms. By abuse of notation, we denote the resulting maps with the same symbol $\Gamma_{X^{[n]}}^{[n]}$ :

$$
\begin{aligned}
& \Gamma_{X}^{[n]}: \bigoplus_{t=0}^{2 n}\left(\bigoplus_{i+(n-l(\nu))=t} R_{\nu}^{i}[l(\nu)]\right)[n-t] \stackrel{\simeq}{\longrightarrow} h_{n *} \mathbb{Q}_{X}^{[n]}[n] ; \\
& \Gamma_{X}^{[n]}: \bigoplus_{k=0}^{2 n}\left(\bigoplus_{i+2(n-l(\nu))=k} R_{\nu}^{i}\right)[-k] \stackrel{\simeq}{\longrightarrow} h_{n *} \mathbb{Q}_{X}{ }^{[n]} .
\end{aligned}
$$

We now turn to the decompositions in cohomology stemming from the isomorphism(s) $\Gamma_{X}^{[n]}$. By taking components in (10), we have the equality of maps in the derived category

$$
\Gamma_{X}^{[n]}=\sum_{\nu} \Gamma_{X}^{(\nu)}=\sum_{\nu} \sum_{i=0}^{2 l(\nu)} \Gamma_{X}^{(\nu), i}
$$

and, by taking the images in cohomology, we set

$$
G_{\nu}^{*}\left(X^{[n]}, \mathbb{Q}\right):=\operatorname{Im} \Gamma_{X}^{(\nu)} \subseteq H^{*}\left(X^{[n]}, \mathbb{Q}\right) .
$$

By the very construction of the splitting (10), i.e. the fact that is it obtained by pushing forward (1), we have that

$$
G_{\nu}\left(X^{[n]}, \mathbb{Q}\right): \stackrel{13}{=} \operatorname{Im} \Gamma_{X}^{(\nu)}=\operatorname{Im} \gamma_{X}^{(\nu)} \stackrel{\underline{\underline{3}}}{=}: H_{\nu}^{*}\left(X^{[n]}, \mathbb{Q}\right) \subseteq H^{*}\left(X^{[n]}, \mathbb{Q}\right)
$$

or, in words, the two distinguished splittings of $H^{*}\left(X^{[n]}, \mathbb{Q}\right)$ into $\nu$-components arising from the decomposition theorem for the Hilbert-Chow map $\pi_{n}$ and for the Hitchin-like map $h_{n}$ coincide.

For every fixed partition $\nu$ of $n$ and for every $0 \leq i \leq 2 l(\nu)$, we set

$$
H_{\nu, i}^{*}\left(X^{[n]}, \mathbb{Q}\right):=\operatorname{Im} \Gamma_{X}^{(\nu), i} \subseteq H_{\nu}^{*}\left(X^{[n]}, \mathbb{Q}\right),
$$

so that

$$
H_{\nu}^{*}\left(X^{[n]}, \mathbb{Q}\right)=\bigoplus_{i=0}^{2 l(\nu)} H_{\nu, i}^{*}\left(X^{[n]}, \mathbb{Q}\right)
$$

The following lemma shows that in each cohomological degree $d$, there is at most one non zero summand $H_{\nu, i}^{d}\left(X^{[n]}, \mathbb{Q}\right)$ in (14). 
Lemma 3.2.2 We have the following

$$
H^{q}\left(\mathbb{C}^{(n)}, R_{\nu}^{i}\right) \simeq \begin{cases}0 & \text { if } q \neq 0 \\ H^{i}\left(E^{(\nu)}, \mathbb{Q}\right) & \text { if } q=0\end{cases}
$$

In particular, for every d, we have that

$$
H_{\nu}^{d}\left(X^{[n]}, \mathbb{Q}\right)=H_{\nu, i=d-2(n-l(\nu))}^{d}\left(X^{[n]}, \mathbb{Q}\right) \simeq H^{d-2(n-l(\nu))}\left(E^{(\nu)}, \mathbb{Q}\right) .
$$

Proof. We have

$$
H^{q}\left(\mathbb{C}^{(n)}, r_{\mathbb{C} *}^{(\nu)}\left(R^{i} p_{*}^{l(\nu)} \mathbb{Q}\right)^{\mathfrak{S}_{\nu}}\right)=H^{q}\left(\mathbb{C}^{(\nu)},\left(R^{i} p_{*}^{l(\nu)} \mathbb{Q}\right)^{\mathfrak{S}_{\nu}}\right)=H^{q}\left(\mathbb{C}^{l(\nu)}, R^{i} p_{*}^{l(\nu)} \mathbb{Q}\right)^{\mathfrak{S}_{\nu}}
$$

Since $\mathbb{C}^{l(\nu)}$ is contractible, the groups above are zero whenever $q \neq 0$. In view of (9), for $q=0$ we have:

$$
H^{0}\left(\mathbb{C}^{l(\nu)},\left(R^{i} p_{*}^{l(\nu)} \mathbb{Q}\right)\right)^{\mathfrak{S}_{\nu}}=H^{i}\left(E^{l(\nu)}, \mathbb{Q}\right)^{\mathfrak{S}_{\nu}}=H^{i}\left(E^{(\nu)}, \mathbb{Q}\right) .
$$

This proves the first statement.

According to (10) and the diagram (8) , each summand $H_{\nu, i}^{d}\left(X^{[n]}, \mathbb{Q}\right)$ is the subspace of $H^{d}\left(X^{[n]}, \mathbb{Q}\right)$ injective image of

$$
\begin{aligned}
H^{d-2(n-l(\nu))-i}\left(\mathbb{C}^{(n)}, r_{\mathbb{C} *}^{(\nu)}\left(R^{i} p_{*}^{l(\nu)} \mathbb{Q}\right)^{\mathfrak{S}_{\nu}}\right) & =H^{d-2(n-l(\nu))-i}\left(\mathbb{C}^{(\nu)},\left(R^{i} p_{*}^{l(\nu)} \mathbb{Q}\right)^{\mathfrak{S}_{\nu}}\right) \\
& =\quad\left(H^{d-2(n-l(\nu))-i}\left(\mathbb{C}^{l(\nu)},\left(R^{i} p_{*}^{l(\nu)} \mathbb{Q}\right)\right)\right)^{\mathfrak{S}_{\nu}}
\end{aligned}
$$

The second statement now follows from (14) and from the first statement.

Summarizing: we have that for every $d$ :

$$
H^{d}\left(X^{[n]}, \mathbb{Q}\right)=\bigoplus_{\nu} H_{\nu}^{*}\left(X^{[n]}, \mathbb{Q}\right)=\bigoplus_{\nu} H_{\nu, d-2(n-l(\nu))}^{d}\left(X^{[n]}, \mathbb{Q}\right) \simeq \bigoplus_{\nu} H^{d-2(n-l(\nu))}\left(E^{(\nu)}, \mathbb{Q}\right) .
$$

\subsection{The perverse Leray filtration $\mathscr{P}_{X^{[n]}}$ on $H^{*}\left(X^{[n]}, \mathbb{Q}\right)$}

The theory of perverse sheaves endows $H^{*}\left(X^{[n]}, \mathbb{Q}\right)$ with the perverse Leray filtration $\mathscr{P}_{X^{[n]}}$, i.e. with the perverse filtration associated with the complex $h_{n *} \mathbb{Q}_{X^{[n]}}[n]$; see [11]. 
Note that if we replace $h_{n *} \mathbb{Q}_{X}^{[n]}[n]$ with another shift $h_{n *} \mathbb{Q}_{X}{ }^{[n]}[m]$, the resulting filtrations gets translated. We have made the choice $m=n$ so that, in view of (11), the result has the same type $[0,2 n]$ as the one of ${ }_{\frac{1}{2}} \mathscr{W}_{Y^{[n]}}$.

While, in general, the perverse (Leray) filtration is canonically defined, there is no natural splitting of it. In our situation, in view of (11) and of (15), we have that the perverse Leray filtration is naturally split:

$$
\mathscr{P}_{X^{[n]}, p} H^{d}\left(X^{[n]}, \mathbb{Q}\right)=\bigoplus_{t \leq p} \bigoplus_{d-(n-l(\nu))=t} H_{\nu}^{d}\left(X^{[n]}, \mathbb{Q}\right)=\bigoplus_{d-(n-l(\nu)) \leq p} H_{\nu}^{d}\left(X^{[n]}, \mathbb{Q}\right) .
$$

Remark 3.3.1 In view of the expression (12), it is straightforward to verify with the aid of Lemma 3.2 .2 that the ordinary Leray filtration $\mathscr{L}_{X^{[n]}}$ on $H^{*}\left(X^{[n]}, \mathbb{Q}\right)$ for the map $h_{n}$ is the filtration by cohomological degree. In particular, by comparing with (16), it is clear that the Leray filtration is strictly included in the perverse Leray filtration.

\section{The main result, relation with hard Lefschetz, and a spec- ulation}

\section{1 $" \mathscr{P}_{X[n]}={ }_{\frac{1}{2}} \mathscr{W}_{Y}{ }^{[n]} "$}

We are now ready to state and prove the main result of this paper.

Theorem 4.1.1 For every $n \geq 0$, the map $\phi^{[n]}$ (7) is a filtered isomorphism, i.e.

$$
\phi^{[n]}\left(\mathscr{P}_{X[n]}\right)={ }_{\frac{1}{2}} \mathscr{W}_{Y^{[n]}} .
$$

Proof. By its very definition, the map $\phi^{[n]}$ is a direct sum map with respect to the $\nu$ decompositions (4) for $S=X$ and $S=Y$, respectively It remains to apply Proposition 3.1 .2 and (16).

We would like to remark on the exceptional circumstance highlighted by Theorem 4.1.1. In view of the canonical splitting (16), we say that a class $a \in H^{d}\left(X^{[n]}, \mathbb{Q}\right)$ has

perversity $p$ if $a \in \oplus_{d-(n-l(\nu))=p} H^{d}\left(X^{[n]}, \mathbb{Q}\right)$. Theorem 4.1.1 shows that, regardless of the $(r, s)$ type of $a$ with respect to the pure Hodge structure $H^{d}\left(X^{[n]}, \mathbb{Q}\right)$, we have that $\phi^{[n]}(a) \in H^{d}\left(Y^{[n]}, \mathbb{Q}\right)$ has type $(p, p)$ and, more precisely, lives in the $(p, p)$ part of the split Hodge-Tate type structure. 
The proof of Theorem 4.1.1 is heavily based on the fact that we have constructed the explicit splitting (16) of the perverse Leray filtration. There is a different approach which is based on the following geometric description [11] of the perverse Leray filtration: let $s \geq 0$ and let $\Lambda^{s} \subseteq \mathbb{C}^{(n)} \simeq \mathbb{C}^{n}$ be a general $s$-dimensional linear section of $\mathbb{C}^{n}$; then

$$
\mathscr{P}_{X^{[n]}, p} H^{d}\left(X^{[n]}, \mathbb{Q}\right)=\operatorname{Ker}\left\{H^{d}\left(X^{[n]}, \mathbb{Q}\right) \longrightarrow H^{d}\left(h_{n}^{-1}\left(\Lambda^{d-p-1}\right), \mathbb{Q}\right)\right\} .
$$

While we omit the details of this approach, we do point out the basic fact leading to the identification of the kernel above with the right-hand-side of (16): a general linear section $\Lambda^{d-p-1}$ avoids the closure of a stratum $\overline{\mathbb{C}_{\nu}^{(n)}}$, which has dimension $l(\nu)$, if and only if $d-(n-l(\nu)) \leq p$.

\subsection{The curious hard Lefschetz $(\mathrm{CHL})$ for $H^{*}\left(\left(\mathbb{C}^{*} \times \mathbb{C}^{*}\right)^{[n]}, \mathbb{Q}\right)$}

Let $(z, w)$ be coordinates on $Y=\mathbb{C}^{*} \times \mathbb{C}^{*}$. The 2 -form

$$
\alpha_{Y}:=\frac{1}{(2 i \pi)^{2}} \frac{d z \wedge d w}{z w}
$$

is closed and defines an integral cohomology class which we denote with the same symbol. We have $\alpha_{Y} \in H^{2}(Y, \mathbb{Q}) \cap H^{2,2}(Y)$. Let $p_{i}: Y^{n} \rightarrow Y$ be the $i$-th projection. Set $\alpha_{Y^{n}}=\sum_{i=1}^{n} p_{i}^{*} \alpha_{Y} \in H^{2}\left(Y^{n}, \mathbb{Q}\right) \cap H^{2,2}\left(Y^{n}\right)$. Let $\alpha_{Y(n)} \in H^{2}\left(Y^{(n)}, \mathbb{Q}\right) \cap H^{2,2}\left(Y^{(n)}\right)$ and $\alpha_{Y^{(\nu)}} \in H^{2}\left(Y^{(\nu)}, \mathbb{Q}\right) \cap H^{2,2}\left(Y^{(\nu)}\right)$ be the naturally induced classes. Let $\alpha_{Y^{[n]}}:=\pi_{n}^{*} \alpha_{Y^{(n)}} \in$ $H^{2}\left(Y^{(n)}, \mathbb{Q}\right) \cap H^{2,2}\left(Y^{(n)}\right)$ be the pullback via the Hilbert-Chow map $\pi_{n}: Y^{[n]} \rightarrow Y^{(n)}$.

Note that because of Hodge type, none of the $\alpha$-type classes above is the first Chern class of a holomorphic line bundle on $Y^{[n]}$. Nonetheless, a simple explicit computation based on Proposition 3.1.2 shows that cupping with the powers of $\alpha_{Y^{[n]}}$, gives rise to isomorphisms

$$
\operatorname{Gr}_{2 n-2 k}^{\mathscr{W}_{Y}[n]} H^{*}\left(Y^{[n]}, \mathbb{Q}\right) \underset{Y_{Y[n]}^{k}}{\simeq} \operatorname{Gr}_{2 n+2 k}^{\mathscr{W}_{Y}^{[n]}} H^{*+2 k}\left(Y^{[n]}, \mathbb{Q}\right)
$$

These isomorphisms are analogous to the "curious hard Lefschetz" theorem of [17. Its curiosity consists of the fact that it is a statement concerning a $(2,2)$ class on a noncompact variety, instead of a $(1,1)$-class on a projective variety. This apparently mysterious fact receives an explanation from the coincidence of the halved weight filtration with the perverse Leray filtration proved in the main Theorem 4.1.1.

Question 4.2.1 What corresponds to the CHL (17) under the identification $H^{*}\left(Y^{[n]}, \mathbb{Q}\right) \simeq$ $H^{*}\left(X^{[n]}, \mathbb{Q}\right)$ given by $(7)$ ? We answer this question in Theorem 4.3.2, 


\subsection{CHL on $Y^{[n]} \Leftrightarrow$ the HL on $E^{(\nu)} \Leftrightarrow$ RHL for $h_{n}$}

In this section, we say that a rational cohomology class of degree two on a variety $Z$ is good (resp. ample) if it is a non-zero (resp. positive) rational multiple of the Chern class of an ample line bundle on $Z$. The point of this definition is that the hard Lefschetz theorem holds for a good class on a nonsingular projective manifold as well as on its quotients by a finite group acting by algebraic isomorphisms.

Fix any diffeomorphism $\Phi: Y=\mathbb{C}^{*} \times \mathbb{C}^{*} \simeq X=E \times \mathbb{C}$. We obtain the linear isomorphism (7) of graded vector spaces: $\phi_{[n]}: H^{*}\left(X^{[n]}, \mathbb{Q}\right) \simeq H^{*}\left(Y^{[n]}, \mathbb{Q}\right)$.

Let $\alpha_{X}, \alpha_{X^{n}}, \alpha_{X^{(n)}}, \alpha_{X^{(\nu)}}, \alpha_{X^{[n]}}$ be the classes obtained by transplanting the $\alpha$-classes defined starting from $Y$ in section 4.2 via $\phi_{[n]}^{-1}$.

Note that by construction, for every surface $S$, the inclusion $H^{*}\left(S^{(n)}, \mathbb{Q}\right) \subseteq H^{*}\left(S^{[n]}, \mathbb{Q}\right)$ is given by the pull-back $\pi_{n}^{*}$ via the Hilbert-Chow map $\pi_{n}: S^{[n]} \rightarrow S^{(n)}$. In particular, we have that $\alpha_{X^{[n]}}=\pi_{n}^{*} \alpha_{X^{(n)}}$. This has to be verified in view of the fact that $\phi_{[n]}$ has not been defined using a diffeomorphism $Y^{[n]} \simeq X^{[n]}$ between the Hilbert schemes.

Note that $\phi_{[n]}$ is not a map of MHS (this is already apparent for $n=1$ ). On the other hand, since $H^{2}(X, \mathbb{C})=H^{2}(E \times \mathbb{C}) \simeq H^{2}(E, \mathbb{C})=H^{1,1}(E)$, we see that all the $\alpha$-classes $\alpha_{X}, \ldots, \alpha_{X}[n]$ are in fact in $H^{2}(-, \mathbb{Q}) \cap H^{1,1}(-)$.

Moreover, the class $\alpha_{X} \in H^{2}(X, \mathbb{Q}) \simeq \mathbb{Q}$, being non-zero, is automatically good. In fact, it is ample if and only if the diffeomorphism $\Phi: Y \simeq X$ preserves the canonical orientations of the complex analytic surfaces.

It follows that the $\alpha$-classes $\alpha_{X}, \alpha_{X^{n}}, \alpha_{X^{(n)}}$ and $\alpha_{X^{(\nu)}}$ are good. Since $\alpha_{X^{(\nu)}}$ is good, so is its restriction to the fibers of $X^{(\nu)} \rightarrow \mathbb{C}^{(\nu)}$. The fibers of this map over points in the dense open stratum of $\mathbb{C}^{(\nu)}$ consisting of multiplicity-free cycles are isomorphic to the product $E^{l(\nu)}$. Over the remaining points, the fibers are isomorphic to finite quotients $E^{l(\nu)} / G$, where the $G$ are suitable subgroups of $\mathfrak{S}_{\nu}$ (see section 2.2).

On the other hand, if $n \geq 2$, then $\alpha_{X^{[n]}}$ is not good: being a pull-back from $X^{(n)}$, it is trivial on the positive dimensional projective fibers of the Hilbert Chow birational map $\pi_{n}: X^{[n]} \rightarrow X^{(n)}$, a fact that prohibits goodness.

In view of the identifications of Lemma 3.2 .2 and of the fact that $\alpha_{X^{(\nu)}}$ and its restriction to $E^{(\nu)}$ are good, we have that the classical hard Lefschetz isomorphisms for the nonsingular projective $E^{(\nu)}$ of dimension $l(\nu)$ reads as follows

$$
\alpha_{X^{(\nu)}}^{j}: H_{\nu}^{l(\nu)-j}\left(X^{[n]}, \mathbb{Q}\right)=H^{l(\nu)-j}\left(E^{(\nu)}, \mathbb{Q}\right) \stackrel{\simeq}{\longrightarrow} H^{l(\nu)+j}\left(E^{(\nu)}, \mathbb{Q}\right)=H_{\nu}^{l(\nu)+j}\left(X^{[n]}, \mathbb{Q}\right) .
$$


Remark 4.3.1 Since $\alpha_{X^{[n]}}$ is a pull-back from $X^{(n)}$, its action via cup product on $\pi_{n *} \mathbb{Q}_{X[n]}[2 n]$ is diagonal with respect to the decomposition into $\nu$-summands (11). Moreover, the induced action on each $\nu$-summand is the action via cup product with $\alpha_{X^{(\nu)}}$. The same holds after taking cohomology.

The hard Lefschetz isomorphisms (18) express a property of this cup product action with $\alpha_{X^{[n]}}$ in cohomology. In fact, (18) is the reflection in cohomology of the fact that the conclusion of the relative hard Lefschetz theorem ([1], Theorem 5.4.10; see also [9]) holds for the map $h_{n}: X^{[n]} \rightarrow \mathbb{C}^{(n)}$ and for the cup-product action with $\alpha_{X^{[n]}}$, i.e. that we have isomorphisms

$$
\alpha_{X}^{j}{ }^{[n]}:{ }^{p} \mathcal{H}^{-j}\left(h_{n *} \mathbb{Q}[2 n]\right) \stackrel{\simeq}{\longrightarrow} p_{\mathcal{H}^{j}}\left(h_{n *} \mathbb{Q}[2 n]\right),
$$

where, in view of (10), the perverse cohomology sheaves are

$$
{ }^{p} \mathcal{H}^{j}\left(h_{n *} \mathbb{Q}[2 n]\right)=\bigoplus_{i-l(\nu)=j} R_{\nu}^{i}[l(\nu)]
$$

In fact, the map of perverse sheaves (19) is defined simply because $\alpha_{X^{[n]}} \in H^{2}\left(X^{[n]}, \mathbb{Q}\right)$; see [9]), §4.4. By using the identifications of Lemma 3.2.2, we deduce that the map (19) is an isomorphism: in fact, in view of the isomorphisms (18), it is an isomorphism on the stalks of the respective cohomology sheaves.

Recall that $\alpha_{X^{[n]}}$ is not good for $n \geq 2$, i.e. it is neither "positive", nor "negative" on the fibers of $h_{n}$, so that the relative hard Lefschetz theorem does not apply in this context, yet we have (19). This situation is similar to the one of the paper [6], where the notion of lef line bundles has been introduced and where it is proved that it is strongly linked to the hard Lefschetz theorem. The relation with the present situation is that, up to sign, $\alpha_{X^{[n]}}$ is not ample on the fibers of $h_{n}$, but it is lef.

Recalling the expression (16) for the perverse Leray filtration and Remark 4.3.1, a direct calculation using the hard Lefschetz isomorphisms (18) and Theorem 4.1.1 implies the following result, which answers Question 4.2.1.

Theorem 4.3.2 Under the identification $\phi_{[n]}: H^{*}\left(X^{[n]}, \mathbb{Q}\right)=H^{*}\left(Y^{[n]}, \mathbb{Q}\right)$, the $C H L$ (17) becomes the (relative) hard Lefschetz (19).

We conclude this section by remarking that the splitting (10) of $h_{n *} \mathbb{Q}_{X^{[n]}}$ has a remarkable property. Deligne's paper [13] implies that once we have the relative hard Lefschetztype isomorphisms (19), we can construct three a priori distinct isomorphisms between the l.h.s and the r.h.s of (10). Each one of these three splittings is characterized by a certain property of the matrices that express the action of the cup product operations 
$\alpha_{X[n]}^{k}: h_{n *} \mathbb{Q}_{X}^{[n]} \rightarrow h_{n *} \mathbb{Q}_{X^{[n]}}[2 k]$ with respect to the splitting; see [13], p.118 for the definition of this matrix, Proposition 2.7 for the first splitting, section 3.1 for the second, and Proposition 3.5 for the third. In general, these three splittings differ from each other, e.g. in the case of the projectivization of a vector bundle with non trivial Chern classes, projecting over the base.

In our situation, there is the fourth splitting (10). The remarkable fact is that, in view of Remark 4.3.1, it is a matter of routine to verify that the four splittings coincide.

\subsection{Speculating on where to find the exchange of filtrations}

The example treated in this paper and the one considered in 3 . have some properties in common which lead us to conjecture that the exchange of filtration occur for a certain class of varieties and maps. Let us recall the main theorem of [3]:

Consider the moduli space of semistable Higgs bundles $\mathcal{M}_{\text {Dol }}$ parametrizing stable rank 2 Higgs bundles $(E, \phi)$ of degree 1 on a fixed nonsingular projective curve $C$ of genus $g \geq 2$. There is the Hitchin proper and flat map $h: \mathcal{M}_{\mathrm{Dol}} \longrightarrow \mathbb{C}^{4 g-3}$, which gives rise to the perverse Leray filtration $\mathscr{P}_{\mathcal{M}_{\text {Dol }}}$. By the non-Abelian Hodge theorem, $\mathcal{M}_{\text {Dol }}$ is naturally diffeomorphic to the twisted character variety

$$
\mathcal{M}_{\mathrm{B}}:=\left\{A_{1}, B_{1}, \ldots, A_{g}, B_{g} \in \mathrm{GL}_{2}(\mathbb{C}) \mid A_{1}^{-1} B_{1}^{-1} A_{1} B_{1} \ldots A_{g}^{-1} B_{g}^{-1} A_{g} B_{g}=-\mathrm{I}\right\} / \mathrm{GL}_{2}(\mathbb{C})
$$

where the quotient is taken in the sense of invariant theory. The twisted character variety $\mathcal{M}_{\mathrm{B}}$ carries a natural structure of nonsingular complex affine variety, with Hodge structure of Hodge-Tate type, with a natural splitting.

In complete analogy with Theorem 4.1.1, we have the main result in [3], Theorem 4.2.9

Theorem 4.4.1 In terms of the isomorphism $H^{*}\left(\mathcal{M}_{\mathrm{B}}\right) \stackrel{\simeq}{\longrightarrow} H^{*}\left(\mathcal{M}_{\text {Dol }}\right)$ induced by the diffeomorphism $\mathcal{M}_{\mathrm{B}} \stackrel{\simeq}{\longrightarrow} \mathcal{M}_{\text {Dol }}$ stemming from the non-Abelian Hodge theorem, we have

$$
\mathscr{W}_{\mathcal{M}_{B}, 2 k} H^{*}\left(\mathcal{M}_{\mathrm{B}}\right)=\mathscr{W}_{\mathcal{M}_{B}, 2 k+1} H^{*}\left(\mathcal{M}_{\mathrm{B}}\right)=\mathscr{P}_{\mathcal{M}_{\text {Dol }}, k} H^{*}\left(\mathcal{M}_{\mathrm{Dol}}\right) .
$$

The varieties $\mathcal{M}_{\text {Dol }}$ and $X^{[n]}$ belong to the following class of varieties $Z$ :

1. $Z$ is a quasi-projective nonsingular variety of even dimension $2 m$ endowed with a holomorphic symplectic structure $\omega \in H^{0}\left(Z ; \Lambda^{2} T^{*} Z\right)$ and with a $\mathbb{C}^{*}$-action $\phi$ : $\mathbb{C}^{*} \times Z \rightarrow Z$, such that for $\phi_{\lambda}^{*} \omega=\lambda \omega$ for $\lambda \in \mathbb{C}^{*}$. 
2. The ring $\Gamma\left(Z, \mathcal{O}_{Z}\right)$ is finitely generated and the affine reduction map $h_{Z}: Z \longrightarrow A=$ $\operatorname{Spec} \Gamma\left(Z, \mathcal{O}_{Z}\right)$ is proper with fibres of dimension $m$.

3. The induced action on $A$ has a unique fixed point $o$ such that $\lim _{t \rightarrow 0} t y=o$ for all $y \in A$.

Let us note that, under these hypotheses, the Hodge structure on the cohomology groups $H^{d}(Z, \mathbb{Q})$ is pure of weight $d$ : the inclusion $h^{-1}(o) \subset Z$ induces an isomorphism $H^{d}(Z, \mathbb{Q}) \simeq H^{d}\left(h^{-1}(o), \mathbb{Q}\right)$ of MHS; since $Z$ is nonsingular, the weight inequalities ([12] Theorem 8.2.4, iii. and iv.) imply the purity of $H^{*}(Z, \mathbb{Q})$.

Additionally we see that if $f$ and $g$ are functions in $\Gamma\left(A, \mathcal{O}_{A}\right) \cong \Gamma\left(Z, \mathcal{O}_{Z}\right)$ then we can write them as $f=\sum_{i>0} f_{i}$ and $g=\sum_{i>0} f_{i}$ and $g=\sum_{i>0} g_{i}$ such that $\phi_{\lambda}^{*}\left(f_{i}\right)=\lambda^{i} f_{i}$ and $\phi_{\lambda}^{*}\left(g_{i}\right)=\lambda^{i} g_{i}$. Then the Poisson bracket satisfies

$$
\{f, g\}=\sum_{i, j>0}\left\{f_{i}, g_{j}\right\}=\sum_{i, j>0} \frac{1}{\lambda}\left\{\phi_{\lambda}^{*} f_{i}, \phi_{\lambda}^{*} g_{j}\right\}=\sum_{i, j>0} \lambda^{i+j-1}\left\{f_{i}, g_{j}\right\} .
$$

Because $\lambda^{k} h=h$ for $k>0$ and generic $\lambda \in \mathbb{C}^{*}$ only for the zero function, thus we can conclude $\{f, g\}=0$. Thus $h_{Z}$ is a completely integrable system.

The two examples given in this paper and in 3] lead us to speculate whether it is possible to associate with every variety $Z$ satisfying the three assumptions above another variety $\widetilde{Z}$ such that:

1. $\widetilde{Z}$ is a quasi projective nonsingular variety endowed with a holomorphic symplectic structure.

2. The affine reduction map $h_{\widetilde{Z}}: \widetilde{Z} \longrightarrow \operatorname{Spec} \Gamma\left(\widetilde{Z}, \mathcal{O}_{\widetilde{Z}}\right)$ is birational (hence semismall in view of [19], Lemma 2.11).

3. There is a natural isomorphism $\phi: H^{*}(Z, \mathbb{Q}) \simeq H^{*}(\widetilde{Z}, \mathbb{Q})$.

4. The cohomology groups $H^{*}(\widetilde{Z}, \mathbb{Q})$ have a Hodge structure of split Hodge-Tate type.

5. Under the isomorphism $\phi$, the perverse filtration on $Z$ associated with the map $h$ corresponds to the halved weight filtration on $H^{*}(\widetilde{Z}, \mathbb{Q})$ : a class of perversity $p$ on $Z$ would correspond to a class of type $(p, p)$ on $\widetilde{Z}$.

Let us remark that, if the above were true, then the Hodge structure of $\widetilde{Z}$ cannot be pure. In fact, in view of the relative hard Lefschetz theorem, the class $\alpha \in H^{2}(Z, \mathbb{Q})$ of any $h$-ample class on $Z$ has necessarily perversity 2 . It would then follows that $\phi(\alpha) \in H^{2}(\widetilde{Z}, \mathbb{Q})$ 
would have type $(2,2)$. In view of the conditions we have imposed on the affine reduction maps of the two varieties, i.e. the fact that $h_{Z}$ is a fibration with middle dimensional

fibers and $h_{\widetilde{Z}}$ is semismall, we like to think that $Z$ is "as complete as possible," whereas $\widetilde{Z}$ is "as affine as possible."

At present, we do not know how to attack such a question and we still do not know how to formulate a principle that would justify the exchange of filtrations.

\section{References}

[1] A.A. Beilinson, J.N. Bernstein, P. Deligne, Faisceaux pervers, Astérisque 100, Paris, Soc. Math. Fr. 1982.

[2] M.A. de Cataldo, "The Hodge theory of character varieties," to appear in the Proceedings of the conference Classical Algebraic Geometry Today, M.S.R.I. Berkeley, January 25-29, 2009. arXiv:1011.0784.

[3] M.A. de Cataldo, T. Hausel, L. Migliorini, "Topology of Hitchin systems and Hodge theory of character varieties: the case $A_{1}, "$ Preprint arXiv: 104.1420.

[4] M. A. de Cataldo, L. Migliorini, "The Douady space of a complex surface," Adv. Math. 151 (2000), 283-312.

[5] M.A. de Cataldo, L. Migliorini, "The Chow groups and the motive of the Hilbert scheme of points on a surface," J. Algebra 251 (2002), no. 2, 824-848.

[6] M. de Cataldo, L. Migliorini, "The Hard Lefschetz Theorem and the topology of semismall maps," Ann. Scient. Éc. Norm. Sup., 4e srie, t. 35, 2002, 759-772.

[7] M. de Cataldo, L. Migliorini, "The Chow motive of semismall resolutions," Math. Res. Lett. 11 (2004), 151-170.

[8] M.A. de Cataldo, L. Migliorini, "The Gysin formalism is compatible with mixed Hodge structures," Proceedings volume of the CRM Workshop on Algebraic Structures and Moduli Spaces. AMS-CRM Proceedings series vol. 38,(2004) 133-138.

[9] M.A. de Cataldo, L. Migliorini, "The Hodge Theory of Algebraic maps," Ann. Scient. Éc. Norm. Sup., $4^{e}$ série, t. 38, (2005), 693-750.

[10] M.A. de Cataldo and L. Migliorini, "The Decomposition Theorem and the topology of algebraic maps," Bull. of the Amer. Math. Soc., Vol. 46, n.4, (2009), 535-633. 
[11] M. de Cataldo, L. Migliorini, "The perverse filtration and the Lefschetz Hyperplane Section Theorem," Ann. of Math. 171 n.3 (2010) 2089-2113.

[12] P. Deligne, “ Théorie de Hodge III", Publ. Math., I. H. E. S, 44 (1974), 5-77.

[13] P. Deligne, "Décomposition dans la catégorie dérivée, Motives (Seattle, WA, 1991), 115-128, Proc. Symp. Pure Math., 55, PArt 1, Amer. Math. Soc., Providence, R.I., 1994.

[14] A. Dimca, Sheaves in topology, Universitext. Springer-Verlag, Berlin, 2004.

[15] L. Göttsche, Hilbert schemes of Zero-Dimensional Subschemes of Smooth Varieties, Lecture Notes in Math, vol.1572, Springer Verlag, Berlin, 1994.

[16] L. Göttsche, W. Soergel, "Perverse Sheaves and the cohomology of the Hilbert schemes of smooth algebraic surfaces," Math. Ann. 196(1993), 235-245.

[17] T. Hausel, F. Rodrigues-Villegas, "Mixed Hodge polynomials of character varieties," Invent. Math. 174, No. 3, (2008), 555-624.

[18] A. Iarrobino, Punctual Hilbert schemes Mem. Amer. Math. Soc. 1881977.

[19] D. Kaledin "Symplectic singularities form the Poisson point of view," J. reine angew. Math. 600(2006), 135-156.

[20] M. Kashiwara, P. Schapira, Sheaves on manifolds, Grundlehren der mathematischen Wissenschaften. Vol. 292, Springer-Verlag, Berlin Heidelberg 1990.

[21] B. Iversen, Cohomology of Sheaves, Universitext, Springer-Verlag, Berlin Heidelberg 1986.

[22] H. Matsumura, Commutative ring theory, Cambridge Studies in Advanced Mathematics 8, CUP, Cambridge 1986.

[23] H. Nakajima, Lectures on the Hilbert Schemes of points on surfaces, University Lecture Series, vol.18, Amer.Math.Soc. Providence, RI,1988.

[24] C. A. M. Peters, J. H. M. Steenbrink, Mixed Hodge Structures Ergebnisse der Mathematik und ihrer Grenzgebiete 3. folge, Vol. 52 Springer-Verlag, Berlin Heidelberg 2008.

[25] C. Voisin, "On the Hilbert scheme of points of an almost complex fourfold," Ann. Inst. Fourier (Grenoble) 50 (2000), no. 2, 689-722. 